\title{
Classification of Topological Insulators and Superconductors
}

\author{
Andreas P. Schnyder*, Shinsei Ryu ${ }^{\dagger}$, Akira Furusaki** and Andreas W. W. Ludwig ${ }^{\ddagger}$ \\ *Kavli Institute for Theoretical Physics, University of California, Santa Barbara, CA 93106, USA \\ ${ }^{\dagger}$ Department of Physics, University of California, Berkeley, CA 94720, USA \\ ${ }^{* *}$ Condensed Matter Theory Laboratory, RIKEN, Wako, Saitama 351-0198, Japan \\ ${ }^{*}$ Department of Physics, University of California, Santa Barbara, CA 93106, USA
}

\begin{abstract}
An exhaustive classification scheme of topological insulators and superconductors is presented. The key property of topological insulators (superconductors) is the appearance of gapless degrees of freedom at the interface/boundary between a topologically trivial and a topologically non-trivial state. Our approach consists in reducing the problem of classifying topological insulators (superconductors) in $d$ spatial dimensions to the problem of Anderson localization at a $(d-1)$ dimensional boundary of the system. We find that in each spatial dimension there are precisely five distinct classes of topological insulators (superconductors). The different topological sectors within a given topological insulator (superconductor) can be labeled by an integer winding number or a $Z_{2}$ quantity. One of the five topological insulators is the "quantum spin Hall" (or: $Z_{2}$ topological) insulator in $d=2$, and its generalization in $d=3$ dimensions. For each dimension $d$, the five topological insulators correspond to a certain subset of five of the ten generic symmetry classes of Hamiltonians introduced more than a decade ago by Altland and Zirnbauer in the context of disordered systems (which generalizes the three well known "Wigner-Dyson" symmetry classes).
\end{abstract}

Keywords: Topological phase, Quantum Hall effects, Anderson localization PACS: 73.43.-f, 74.20.Rp, 67.30.H-, 72.25.Dc

\section{A. INTRODUCTION}

We will give a review of the (exhaustive) classification scheme of topological insulators (or: superconductors, 1 presented in Ref. [1]. We can think of topological insulators (superconductors) as being gapped states (thus "insulators") in $d$ spatial dimensions (we consider here $d=1,2,3$ ) with the following property: if we terminate the topological insulator (superconductor) against a "topologically trivial" state, such as e.g. simply vacuum, gapless degrees of freedom will necessarily appear at the interface ("boundary") between the topologically trivial and the topologically non-trivial states - see Fig.1 (We will present some simple, and well known examples shortly.) Moreover, the so-appearing gapless boundary degrees of freedom are completely robust to perturbations. For example, we may subject the topological insulator (superconductor) to arbitrary random potentials or perturbations no matter how strong, without destroying the "gaplessness" of the boundary degrees of freedom, as long as these perturbations do not close the bulk gap and preserve the generic symmetries of the system

\footnotetext{
1 In this work we consider topological insulators (superconductors) without interactions. Since these are gapped states in the $d$-dimensional bulk, such states will be stable to sufficiently weak interactions. However, under what conditions certain different topological states are adiabatically connected when interactions are included is, to a large extent, an open problem to-date.
}

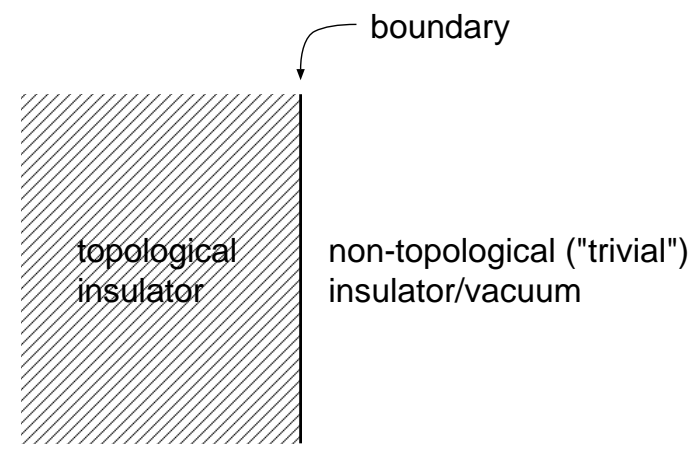

FIGURE 1. Interface between a topological, and a topologically trivial insulator.

(what is meant by these symmetries will be made precise below, and is indeed of fundamental importance in our work). Clearly, these gapless modes must be of a very special kind, since typically, gapless degrees of freedom tend to become localized in the presence of random potentials, certainly if the latter are sufficiently strong (this is the phenomenon of "Anderson localization" for noninteracting systems).

In short, the approach used in this work to classify topological insulators (superconductors) in $d$ spatial dimensions consists in classifying gapless systems of fermions (corresponding to the boundary degrees of freedom) which cannot be localized by disorder. Thus, we reduce the problem of classifying topological insula- 

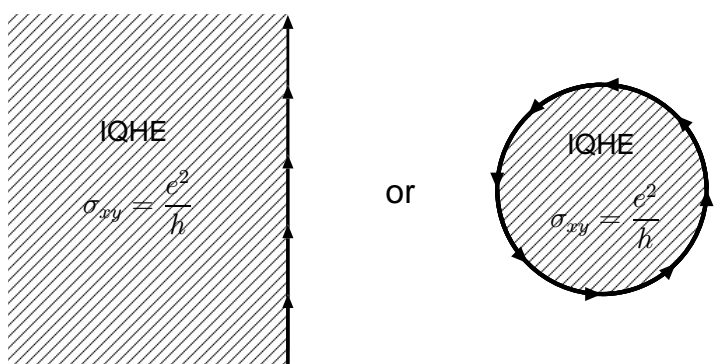

FIGURE 2. Chiral edge states of the integer quantum Hall insulators.

tors (superconductors) in $d$ spatial dimensions to a problem of Anderson localization in $(d-1)$ dimensions. In this work we solve this problem of Anderson localization, and thereby the classification problem for topological insulators (superconductors).

Topological insulators (superconductors) are inherently "holographic" states: the nature of the $d$ dimensional gapped topological bulk state can be read off from the (holographic) "image" or "shadow" of these topological properties on the system's boundaries. Indeed, there is a one-to-one correspondence between the topological properties of the gapped bulk and properties of the gapless surface degrees of freedom. These notions are of course familiar from the quantum Hall effect $\mathrm{I}^{2}$ and it will be useful to remind the reader of (simple) well known examples of such quantum states.

Well known examples of topological insulators (superconductors):

(i): The probably best known example of a topological insulator is the integer quantum Hall insulator (i.e., a filled Landau level). In this non-interacting $d=2$ electron system time-reversal symmetry (TRS) is broken due to the applied magnetic field. If we terminate the quantum Hall insulator by a one-dimensional boundary against "vacuum", a gapless edge state is known to appear (see Fig. 2). This edge state possesses a chirality inherited from the applied magnetic field (broken TRS), and propagates only in one direction; therefore it cannot be localized by disorder.

(ii): Another example in $d=2$ is the chiral $p_{x}+i p_{y}$ superconductor (see e.g. [2]). This is a gapped superconductor, which also breaks TRS. The non-interacting system in question is the system of quasi-particle excitations deep inside the superconducting state, as described by the Bogoliubov-de Gennes (BdG) equation. This is an example of a topological superconductor, as can be seen by terminating the chiral $p_{x}+i p_{y}$ state against vacuum (or an otherwise structureless "standard" supercon-

2 See e.g. [3, 4, 5]; see also [6] for a different context. ducting state): again, at the interface (boundary) a chiral edge mode is known to occur, which, since it propagates only in one direction cannot be localized by disorder [just as that of example (i)]. However, since charge is not a conserved quantity in a superconductor, this chiral edge mode only transports heat (energy) and not charge. This makes clear that while also being a topological gapped state in $d=2$, the chiral $p_{x}+i p_{y}$ superconductor possesses different "symmetries" than the $d=2$ integer quantum Hall state in example (i). (This notion of "symmetries" will be made precise below.)

(iii): Another topological insulating state, often referred to as the $Z_{2}$-topological insulator [7, 8], or the "quantum spin Hall" (QSH) state, has recently attracted much attention. This state is known to exist in $d=2$ and in $d=3$ dimensions and, as opposed to the previous two examples, does not break TRS. It is known to occur in certain band insulators with strong spin-orbit interactions. Let us first discuss the $d=2$ case, realized experimentally e.g. in $\mathrm{HgTe} /(\mathrm{Hg}, \mathrm{Ce}) \mathrm{Te}$ semiconductor quantum wells [9]. Because TRS is not broken, it is not as obvious as in examples (i) and (ii) why the gapless boundary degrees of freedom appearing at the interface terminating the $d=2 Z_{2}$-topological insulator against vacuum cannot be localized by disorder. However, this edge state consists of a single Kramers doublet corresponding to a single pair of modes propagating in opposite directions (see Fig. 3), which cannot be mixed by any TRS impurity potential $3^{3}$ The $Z_{2}$-topological insulator [7], or the QSH state, is also known to exist in $d=3$ dimensions [10, 11, 12]. It is realized in Bismuth-Antimony alloys, as demonstrated in recent experiments [13, 14].

In this work, we ask ourselves the question: which $d$ dimensional (non-interacting) fermion systems possess gapped ground states with topologically non-trivial properties, i.e., which systems are "topological insulators (superconductors)", as described above? How many "different" such systems are there? Are there infinitely many, or only a finite number of them? How do these properties depend on the spatial dimensionality $d$ ? Is there any systematics underlying these different systems?

The answer to these questions turns out to be both deep and interesting: in every spatial dimension $d=1,2,3$ there exist precisely five different classes of topological

\footnotetext{
${ }^{3}$ Indeed, a one-dimensional extended (not localizing) state had already been observed [15] in studies of (quasi 1D) Anderson localization problems with spin-orbit scattering in 1992, but this observation was not understood until recently: truly quasi one-dimensional systems with spin-orbit scattering must always possess two of Kramers doublets which indeed are not protected from localization by disorder; however, when the one-dimensional system is the boundary of what is known today as a two-dimensional $Z_{2}$-topological insulator, a pair of edge states which form a single Kramers doublet appears on each boundary, and such a pair evades Anderson localization.
} 


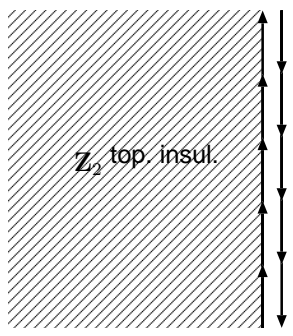

FIGURE 3. Chiral edge states of the $Z_{2}$ topological insulator ("quantum spin Hall effect") in $d=2$ dimensions.

insulators (superconductors). In $d=3$ dimensions, for example, there exist besides the $Z_{2}$-topological insulator discussed in example (iii) above, four more, and all five topologically non-trivial states possess TRS (in $d=3$ ). Our results are summarized in Table 2 In order to explain Table 2, we first need to explain the very general symmetry classification of quantum mechanical Hamiltonians, and explain why this is a fundamental concept underlying the classification scheme of topological insulators (superconductors). This will be done in the following section, Section B. In section $\mathrm{C}$ we will explain with a few very simple examples how topology can arise in simple systems such as band insulators. In section D we will describe the classification of topological insulators (superconductors) in $d=3$ spatial dimensions. Sections $\mathrm{E}$ and $\mathrm{F}$ will provide a summary of the same classification in $d=2$ and $d=1$ dimensions. Section $\mathrm{G}$ provides a discussion and concluding remarks.

Our work [1] demonstrates an unexpected relationship between two apparently rather unrelated subjects. One is the symmetry classification of general quantum mechanical Hamiltonians ("The 10-fold Way" to be reviewed in Section B below), which is at the root of theories of disordered systems, be it Anderson localization, or random matrix theory 4 The other is the classification of topological insulators. It is rather surprising, initially, that a relationship exists between these two subjects. Moreover, a relationship with a third subject has recently emerged. Recent work by A. Kitaev reached [16], by using Ktheory, the same conclusions as those obtained in [1] for the classification of topological insulators. Thus there are remarkable connections between the seemingly rather disjoint subjects of (i) topological insulators, (ii) Anderson localization, random systems, and random matrix theory, as well as (iii) K-theory.

\footnotetext{
4 This classification appeared in the work of Zirnbauer [17], and Altland and Zirnbauer [18, 19], more than a decade ago. It extends the well familiar "Wigner Dyson" classification of Hamiltonians ("unitary, orthogonal, symplectic" classes).
}

\section{B. SYMMETRY CLASSIFICATION OF HAMILTONIANS - "THE 10-FOLD WAY"}

Consider the gapped Hamiltonian and the corresponding ground state of a $d$-dimensional topological insulator 5 Because of the presence of the gap, we may deform the Hamiltonian slightly, by adding various perturbations to it (which preserve the generic symmetries such as, e.g., time reversal symmetry), while still preserving the gap. In this way, we map out an entire gapped phase. We may then ask the question: how many different such phases can a system possess, so that in going from one phase to another, a quantum phase transition has to be crossed? Now, clearly, because of the bulk gap, we will also remain in the same phase by perturbing our Hamiltonian by perturbations which break translational invariance (certainly, as long as these perturbations are small enough). The most general such perturbation is what we call a random perturbation. The perturbed Hamiltonian is thus a random (=lacking translational invariance) gapped Hamiltonian. If the original (unperturbed) phase was topological (in the sense described above), then the random Hamiltonian will be in the same (topological) phase. Therefore we see that in attempting to classify topological phases, we need to consider in general random gapped Hamiltonians. Thus a given gapped topological phase is associated with a certain class of gapped random Hamiltonians. Hence we are led to study the classification of random gapped Hamiltonians. How many such Hamiltonians are there? Clearly, in attempting to classify random Hamiltonians, we can only use the "most generic quantum mechanical symmetries", translational invariance not being one of them. The symmetry properties that every quantum mechanical Hamiltonian can be classified by are time-reversal symmetry (TRS) and charge-conjugation (or: "particle-hole") symmetry (PHS). Investigating the properties of a general Hamiltonian under such symmetries yields the now famous ten symmetry classes (the "ten-fold way"), originally described in the seminal work of Zirnbauer [17], and Altland and Zirnbauer [18, 19], more than a decade ago. This classification extends and completes the familiar "three-fold way" classification scheme of Wigner and Dyson [20], going back to the origins of random matrix theory and the study of complex nuclei. The reason why there are only ten possible symmetry classes of quantum mechanical Hamiltonians is easy to understand by considering TRS and PHS. Let us begin with the time-reversal operator $T$ which is an anti-unitary operator. Thus $T$ is the product of a unitary operator $U_{T}$ and

\footnotetext{
${ }^{5}$ Consider the BdG Hamiltonian in the case of the topological superconductor.
} 
TABLE 1. Ten symmetry classes of single particle Hamiltonians classified in terms of the presence or absence of timereversal symmetry (TRS) and particle-hole symmetry (PHS), as well as sublattice (or "chiral") symmetry (SLS) [17, 18, 19]. In the table, the absence of symmetries is denoted by " 0 ". The presence of these symmetries is denoted either by " +1 " or "-1", depending on whether the (anti-unitary) operator implementing the symmetry squares to " +1 "or " -1 ". For the first six entries of the table (which can be realized in non-superconducting systems) TRS $=+1$ when the SU(2) spin is integer and TRS $=-1$ when it is a half-integer. For the last four entries, the superconductor "Bogoliubov-de Gennes" (BdG) symmetry classes (denoted by the symbols D, C, DIII, and CI in "Cartan nomenclature"), it turns out that the Hamiltonian preserves $\mathrm{SU}(2)$ spin-1/2 rotation symmetry when $\mathrm{PHS}=-1$ whilst it does not preserve $\mathrm{SU}(2)$ when $\mathrm{PHS}=+1$. The column entitled "Hamiltonian" lists the spaces to which the quantum mechanical time-evolution operators of each symmetry class belong (see section B). The column entitled "NLSM (ferm. replicas)" lists the "target spaces" of Non-Linear Sigma Model field theories describing Anderson localization physics in each symmetry class (see section B).

\begin{tabular}{|c|c|c|c|c|c|c|}
\hline System & Cartan nomenclature & TRS & PHS & SLS & Hamiltonian & NLSM (ferm. replicas) \\
\hline \multirow{3}{*}{$\begin{array}{c}\text { standard } \\
\text { (Wigner-Dyson) }\end{array}$} & A (unitary) & 0 & 0 & 0 & $U(N)$ & $U(2 n) / U(n) \times U(n)$ \\
\hline & AI (orthogonal) & +1 & 0 & 0 & $U(N) / O(N)$ & $S p(2 n) / S p(n) \times S p(n)$ \\
\hline & AII (symplectic) & -1 & 0 & 0 & $U(2 N) / S p(2 N)$ & $O(2 n) / O(n) \times O(n)$ \\
\hline \multirow{3}{*}{$\begin{array}{c}\text { chiral } \\
\text { (sublattice) }\end{array}$} & AIII (chiral unit.) & 0 & 0 & 1 & $U(N+M) / U(N) \times U(M)$ & $U(n)$ \\
\hline & BDI (chiral orthog.) & +1 & +1 & 1 & $S O(N+M) / S O(N) \times S O(M)$ & $U(2 n) / S p(n)$ \\
\hline & CII (chiral sympl.) & -1 & -1 & 1 & $S p(2 N+2 M) / S p(2 N) \times S p(2 M)$ & $U(2 n) / O(2 n)$ \\
\hline \multirow[t]{4}{*}{$\mathrm{BdG}$} & $\mathrm{D}$ & 0 & +1 & 0 & $S O(2 N)$ & $O(2 n) / U(n)$ \\
\hline & $\mathrm{C}$ & 0 & -1 & 0 & $S p(2 N)$ & $S p(n) / U(n)$ \\
\hline & DIII & -1 & +1 & 1 & $S O(2 N) / U(N)$ & $O(2 n)$ \\
\hline & $\mathrm{CI}$ & +1 & -1 & 1 & $\operatorname{Sp}(2 N) / U(N)$ & $S p(n)$ \\
\hline
\end{tabular}

the "complex conjugation operator" $K$, i.e., $T=K U_{T}$. On the first quantized Hamiltonian $\mathscr{H}$ time reversal acts as $\mathscr{H} \rightarrow U_{T}^{\dagger} \mathscr{H}^{*} U_{T}$. Now, any Hamiltonian can behave in three possible ways under TRS: (i): it is not invariant under TRS, which case we denote by $\mathrm{TRS}=0$, or, (ii): it is invariant under TRS and the (anti-unitary) time reversal operator $T$ squares to +1 , which case we denote by TRS $=+1$, or, (iii) it is invariant under TRS and the (anti-unitary) time reversal operator $T$ squares to -1 , which case we denote by TRS $=-1$. Similarly, it is possible to describe the particle-hole (charge-conjugation) symmetry (PHS) operator as an anti-unitary operator $C$, when acting on a non-interacting system (see Ref. [1] for details). Therefore, analogously, the possible behaviors of any (non-interacting) Hamiltonian under PHS is $\mathrm{PHS}=0,+1,-1$ (meaning that PHS is not a symmetry, or is a symmetry and the anti-unitary operator $C$ squares to +1 or -1 , respectively). It is now easy to see that there are precisely ten symmetry classes (i.e., those found by Zirnbauer and Altland [17, 18, 19]): There are $3 \times 3=9$ different choices for the behavior of any Hamiltonian under TRS and PHS. A moment's thought shows that for 8 of these 9 choices the behavior of the Hamiltonian under the product $\mathrm{SLS}:=T * C$ of TRS and PHS, which is a

\footnotetext{
${ }^{6}$ The so-defined symmetry operation is sometimes called "sublattice symmetry" (or also: "chiral symmetry"), hence the notation SLS, because a particular (and popular) example of this symmetry arises in systems described by a hopping Hamiltonian on a bipartite lattice, where only matrix elements for hopping between the two different sublattices
}

unitary operator, is uniquely fixed. (We write $\mathrm{SLS}=0$ if the operation SLS is not a symmetry of the Hamiltonian, and $\mathrm{SLS}=1$ if it is.) The only case when the behavior under the combined transformation SLS is not uniquely determined by the behavior under TRS and PHS is when $\mathrm{TRS}=0$ and simultaneously $\mathrm{PHS}=0$. In this case either $\mathrm{SLS}=1$ or $\mathrm{SLS}=0$ is possible. This reasoning gives hence $(3 \times 3-1)+2=10$ possible behaviors of a Hamiltonian.

These are the ten symmetry classes mentioned above, which are listed in Table 1. The column "Hamiltonian" describes the nature of the time evolution operator $\exp \{$ it $\mathscr{H}\}$, where $\mathscr{H}$ is the first quantized Hamiltonian. If we consider a discretized version of the system, e.g., on a (finite) lattice (as, e.g., for a hopping Hamiltonian), then the Hamiltonian $\mathscr{H}$ is a finite $N \times N$ matrix 7 In each symmetry class the time evolution operator is an element of the particular group or symmetric space, listed in this column. For example, if the system has no symmetries at all, it belongs to symmetry class A. This is the case for a quantum Hall system, where TRS is broken 8 In this case, there are no (symmetry) constraints

of the bipartite lattice are non-vanishing. However, this symmetry can be viewed generally simply as the product of $T$ and $C$, as stated; we still denote it by the symbol SLS.

${ }^{7} \mathrm{~N}$ is the product of the number of lattice sites, times the number of spin orientations (e.g., spin-up and spin-down), if applicable, etc. ...

${ }^{8}$ The quantum Hall insulator mentioned in example (i) above belongs to symmetry class $\mathrm{A}$. 
on the Hamiltonian and $\mathscr{H}$ is a generic Hermitian matrix. The time-evolution operator is thus a generic unitary matrix (an element of the group $U(N)$ of unitary matrices, as noted in Table 1, without any further conditions imposed. The first three rows in Table 1 denote thus the standard ("Wigner-Dyson") symmetry classes ("unitary, orthogonal, symplectic"); these are distinguished only by the presence or absence of TRS, and possess no other symmetries. Example (iii) discussed above belongs to symmetry class AII, in which the only symmetry is TRS, with the (non-unitary) TRS operator squaring to minus the identity operator.

The next three rows in Table 1 are identical to the first three rows, except that all possess an additional SLS symmetry $(\mathrm{SLS}=1)$, whereas $\mathrm{SLS}=0$ for the first three rows. We will defer discussion of these symmetry classes for now, except that we recall (footnote 6) that examples of simple realizations of all three (AIII, BDI, CII) can be obtained from hopping models where particles only hop between the two sublattices of a bipartite lattice, with the corresponding TRS properties also imposed.

The last four columns describe the symmetry properties of the fermionic quasiparticles in superconductors (or certain superfluids), deep inside the superconducting state, within a mean field treatment of pairing. Their dynamics is described by the BdG Hamiltonian, which is the Hamiltonian $\mathscr{H}$ whose properties are listed in the column entitled "Hamiltonian". Any BdG Hamiltonian possesses by construction a PHS, as indicated in Table 1. Example (ii) of the $d=2$ dimensional $p+i p$ superconductor (of spinless fermions) belongs to symmetry class $\mathrm{D}$ (7th row): the system possesses no symmetries (including TRS) other than the PHS inherent in all BdG Hamiltonians. Let us conclude by pointing out that symmetry class DIII (9th row) describes the superfluid phase of ${ }^{3} \mathrm{He}-\mathrm{B}$ [21], whereas the CI (10th row) describes, e.g., singlet superconductors. Topological phases of the latter, in $d=3$ dimensions, were discussed in the recent paper [22].

Let us finally comment briefly on the last column of Table 1 (An understanding of this column is not required in order to be able to follow the rest of this review.) It refers to the conventional long-wavelength description of Anderson localization of non-interacting fermions subject to disorder potentials, in terms of a Non-Linear Sigma Model (NLSM) field theory. A NLSM can be viewed as a generalization of the classical Heisenberg ferromagnet, described by a model of classical unit vector spins. These spins can sweep out a sphere, the simplest example of what is called a symmetric space. It is known since the days of the mathematician E. Cartan, that there exist only 10 types of symmetric spaces (barring so-called "exceptional" cases). In general, Anderson localization transitions can be formulated in terms of NLSM field theories of generalized spins, which sweep

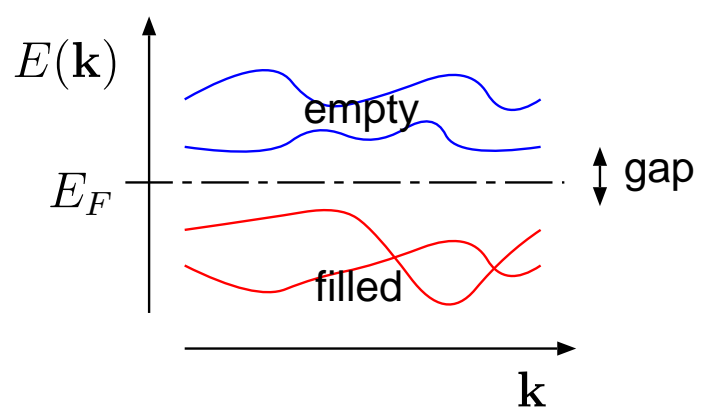

FIGURE 4. Schematic band structure of a typical band insulator.

out one of the 10 symmetric spaces (the "target space"), defining the NLSM. The symmetry class determines which target space is to be used, and these are listed? in the last column of Table 1

\section{THE ORIGIN OF TOPOLOGY IN BAND INSULATORS}

In order to illustrate in the simplest terms how topological properties arise in topological insulators, let us begin with a translationally invariant example. A topological insulator is a very simple system. It is a band insulator of non-interacting fermions, meaning that there is a gap between valence and conduction bands, and the fermi level $E_{F}$ lies in this gap (see Fig. 4). Due to the assumed translational invariance, the insulator is described in momentum space by a matrix equation for every value of momentum $\vec{k}$ in the Brillouin zone

$$
\mathscr{H}(\vec{k})\left|u_{a}(\vec{k})\right\rangle=E_{a}(\vec{k})\left|u_{a}(\vec{k})\right\rangle,
$$

where $a$ denotes an index labeling different bands. Let us now consider, for every momentum $\vec{k}$ in the Brillouin zone, the projection operator onto the filled (Bloch) states,

$$
P(\vec{k}):=\sum_{a}^{\text {filled }}\left|u_{a}(\vec{k})\right\rangle\left\langle u_{a}(\vec{k})\right|
$$

\footnotetext{
9 Specifically, we chose here the simplest formulation in terms of a set of $n$ "fermionic replicas", where $n$ has to be taken to zero at the end. In this formulation the symmetric spaces are all compact, when $n$ is finite. The homotopy group of a symmetric space $\mathscr{M}$ tells us if it is possible to add a topological term $\left(\theta\right.$ term when $\Pi_{d}(\mathscr{M})=\mathbf{Z}$ and $Z_{2}$ term when $\Pi_{d}(\mathscr{M})=\mathbf{Z}_{2}$ ) to a NLSM. A technically better controlled, but equivalent formulation can be provided using a supersymmetric formulation [23], in which the manifolds listed in the last column are replaced by supermanifolds [24], containing compact, non-compact, and fermionic coordinates.
} 
TABLE 2. Summary of the main result of this paper: listed are again the ten symmetry classes of single particle Hamiltonians (from TABLE 1) classified in terms of the presence or absence of time-reversal symmetry (TRS) and particle-hole symmetry (PHS), as well as sublattice (or “chiral”) symmetry (SLS) [17, 18, 19]. The last three columns list all possible topologically non-trivial quantum ground states as a function of symmetry class and spatial dimension $d$. The symbols $\mathbf{Z}$ and $\mathbf{Z}_{2}$ indicate that the space of quantum ground states is partitioned into different topological sectors labeled by an integer $(\mathbf{Z})$, or a $\mathbf{Z}_{2}$ quantity (two sectors only), respectively.

\begin{tabular}{|c|c|c|c|c|c|c|c|}
\hline System & Cartan nomenclature & TRS & PHS & SLS & $d=1$ & $d=2$ & $d=3$ \\
\hline \multirow{3}{*}{$\begin{array}{c}\text { standard } \\
\text { (Wigner-Dyson) }\end{array}$} & A (unitary) & 0 & 0 & 0 & - & $\mathbf{Z}$ & - \\
\hline & AI (orthogonal) & +1 & 0 & 0 & - & - & - \\
\hline & AII (symplectic) & -1 & 0 & 0 & - & $\overline{\mathbf{Z}_{2}}$ & $\overline{\mathbf{Z}_{2}}$ \\
\hline \multirow{3}{*}{$\begin{array}{c}\text { chiral } \\
\text { (sublattice) }\end{array}$} & AIII (chiral unit.) & 0 & 0 & 1 & $\mathbf{Z}$ & - & $\mathbf{Z}$ \\
\hline & BDI (chiral orthog.) & +1 & +1 & 1 & $\mathbf{Z}$ & - & - \\
\hline & CII (chiral sympl.) & -1 & -1 & 1 & $\mathbf{Z}$ & - & $\mathbf{Z}_{2}$ \\
\hline \multirow[t]{4}{*}{$\mathrm{BdG}$} & $\mathrm{D}$ & 0 & +1 & 0 & $\mathbf{Z}_{2}$ & $\mathbf{Z}$ & - \\
\hline & $\mathrm{C}$ & 0 & -1 & 0 & - & $\mathbf{Z}$ & - \\
\hline & DIII & -1 & +1 & 1 & $\mathbf{Z}_{2}$ & $\mathbf{Z}_{2}$ & $\mathbf{Z}$ \\
\hline & $\mathrm{CI}$ & +1 & -1 & 1 & - & - & $\overline{\mathbf{Z}}$ \\
\hline
\end{tabular}

Instead of $P(\vec{k})$ it turns out to be more convenient to use the operator 10

$$
Q(\vec{k}):=\mathbf{1}-2 P(\vec{k})
$$

which has the following properties (as one readily checks)

$$
Q^{\dagger}(\vec{k})=Q(\vec{k}), \quad[Q(\vec{k})]^{2}=\mathbf{1}, \quad \operatorname{tr}[Q(\vec{k})]=m-n,
$$

where $m$ and $n$ denote the number of filled and empty bands, respectively. The Hermitian operator $Q(\vec{k})$ plays the role of the Hamiltonian, carrying only the essential information about the insulator in question. It has eigenvalues \pm 1 . This "simplified Hamiltonian" is obtained from $\mathscr{H}(\vec{k})$ by assigning, say, to all occupied bands the energy -1 and to all empty bands the energy +1 (while all wave functions remain unchanged). Since we are only interested in the properties of the phase described by the insulator, we may deform the actual Hamiltonian of the band insulator until it acquires the simple form $Q(\vec{k})$, while remaining in the same phase.

In order to see how to use the "Hamiltonian" $Q(\vec{k})$, let us begin by considering a band insulator in the simplest symmetry class, in which there are no conditions whatsoever imposed on the Hamiltonian. This is symmetry class A, where the Hamiltonian $\mathscr{H}$ is nothing but a general Hermitian matrix. In this symmetry class, the set of $n+m$ eigenvectors (each being an $n+m$-dimensional vector) forms an arbitrary unitary matrix, i.e., an element of $U(n+m)$. There is however a simple "gauge symmetry", because relabeling the empty and the filled states

$10 \mathbf{1}$ denotes the identity operator. amongst themselves (and forming arbitrary linear combinations amongst them) does not change the physics. Therefore, the Hamiltonian $Q(\vec{k})$ is actually an element of the so-called "Grassmannian"

$$
Q(\vec{k}) \in U(n+m) /[U(n) \times U(m)] .
$$

Since $\vec{k}$ runs over the Brillouin zone $B Z$, the "Hamiltonian" of the band insulator is a map from the Brillouin zone into the Grassmannian,

$$
\begin{aligned}
Q: B Z & \rightarrow U(n+m) /[U(n) \times U(m)], \\
\vec{k} & \rightarrow Q(\vec{k}) .
\end{aligned}
$$

Let us summarize. The Hamiltonian of a band insulator can be continuously deformed to the simple form $Q(\vec{k})$ while remaining in the same phase (i.e., without crossing a quantum phase transition). Now, the question as to how many inequivalent phases there are, amounts to asking how many different maps $Q(\vec{k})$ as in (4) there are which cannot be continuously deformed into each other. This question, on the other hand, is answered 11 by the homotopy group of the map in (4).

Let us consider this in dimensions $d=2$ and $d=3$. In $d=2$ the relevant homotopy group is

$$
\Pi_{2}[U(n+m) /[U(n) \times U(m)]]=\mathbf{Z},
$$

11 Besides the features described by the homotopy group (describing so-called "strong topological" insulators), there are additional features related to the fact that the Brillouin zone is a $d$-dimensional torus. These are so-called "weak topological" features (see [10]) related to the presence of layers of topological insulators [25], i.e., in one dimension less than the space dimension $d$. So-called "weak topological insulators" possess only the latter, by not the former topological features. 
where $\mathbf{Z}$ is the set of all integers. This means that for every integer there exists a band insulator in $d=2$ dimensions in symmetry class $\mathrm{A}$, and band insulators corresponding to different integers cannot be continuously deformed into each other without crossing a quantum phase transition. We have encountered precisely these band insulators already in example (i). These are the quantum Hall insulators, and the integer characterizing the insulator denotes precisely the number of chiral edge states. When the number of edge states changes, a quantum phase transition necessarily has to be crossed. These are precisely the well studied quantum Hall plateau transitions (driven by disorder) 12

Let us now move on to $d=3$ dimensions, still remaining in symmetry class A. Now the relevant homotopy group is (for sufficiently large values of $n$ and $m$ )

$$
\Pi_{3}[U(n+m) /[U(n) \times U(m)]]=\{\mathbf{1}\},
$$

which is the trivial group of only one element, as indicated. This means that here band insulators can only be in one phase. In $d=3$ spatial dimensions there are hence no non-trivial topological insulators in symmetry class A.

Are there then any topologically non-trivial band insulators in $d=3$ dimensions at all? The answer 13 is "yes". We can see this for example from the observation that the presence of SLS is a potential "source" of nontrivial topological behavior. A look at Table 1 reveals that there are five symmetry classes which possess SLS, i.e., which have entries SLS $=1$. (This, as it turns out, does not mean however, that there are non-trivial topological band insulators in all these five symmetry classes.) What is the technical benefit of SLS? It arises from the observation 14 that the presence of this symmetry implies that the Hamiltonian $\mathscr{H}$ can be brought into block offdiagonal form, i.e., that

$$
Q(\vec{k})=\left(\begin{array}{cc}
0 & q(\vec{k}) \\
q^{\dagger}(\vec{k}) & 0
\end{array}\right), \quad \text { where } q(\vec{k}) \text { is unitary. }
$$

Consider now the simplest symmetry class with SLS= 1 , which possesses no symmetry other than SLS. This is symmetry class AIII (4th row of Table 11). Due to the lack of any additional symmetry constraint, $q(\vec{k})$ is an arbitrary unitary matrix, which fully characterizes a

\footnotetext{
12 The field theory describing this transition is the $d=2$ dimensional NLSM on the target space listed for class A in the last column of Table 1 supplemented by a (topological) theta term [26].

13 We have already mentioned in example (iii) of the Introduction (Section A) that there exist in $d=3$ topological insulators in the presence of strong spin-orbit interactions. In the language of Table 1 and Table 2 these belong to symmetry class AII, and will be discussed below.

14 which is easy to check; see [1].
}

phase of the band insulator in this symmetry class. Thus, as in the case of class A considered before, we now need to investigate the homotopy group of maps from the BZ into the group $U(m)$ of unitary matrices. This homotopy group is non-trivial in $d=3$ dimensions,

$$
\Pi_{3}[U(m)]=\mathbf{Z}
$$

This means that in symmetry class AIII there exists a distinct band insulator for every integer, and band insulators characterized by different integers cannot be adiabatically deformed into each other without crossing a quantum phase transition. For completeness, let us also give the explicit form of the integer $v(q(\vec{k}))$, as a functional of $q(\vec{k})$ which characterizes the Hamiltonian:

$$
\begin{gathered}
v(q(\vec{k}))= \\
=\int_{B Z} \frac{d^{3} \vec{k}}{24 \pi^{2}} \varepsilon^{\mu v \rho} \operatorname{tr}\left[\left(q^{-1} \partial_{\mu} q\right)\left(q^{-1} \partial_{\nu} q\right)\left(q^{-1} \partial_{\rho} q\right)\right]
\end{gathered}
$$

where $\varepsilon^{\mu v \rho}$ is the usual totally antisymmetric tensor $\left(\varepsilon^{123}=+1\right)$.

Having presented the appearance of topological properties for band insulators in the two symmetry classes A and AIII, we will now briefly comment on how to extend this to the other classes, even though we will use a different approach to arrive at the classification scheme, to be discussed in the next section. For the (five) symmetry classes with $\mathrm{SLS}=0$, the (simplified) Hamiltonian $Q(\vec{k})$ will satisfy additional conditions. For example, in symmetry class AII [mentioned in example (iii)], the TRS condition has to be imposed which reads

$$
\sigma^{y} Q^{*}(\vec{k}) \sigma^{y}=Q(-\vec{k}) .
$$

Even though in $d=3$ dimensions there existed only a single phase in class A (where $Q(\vec{k})$ was subject to no constraints), the set of all Hamiltonians satisfying the additional constraint (7) turns out to consist of two phases (or sectors) which cannot be continuously deformed into each other. Similarly, for all other symmetry classes with $\mathrm{SLS}=1$, there will be certain constraints on the matrices $q(\vec{k})$, which appeared in (5). For example, in symmetry class CI one turns out to have $q^{t}(-\vec{k})=q(\vec{k})$. A list of these constraints for all ten symmetry classes is provided in Table III of [1].

\section{CLASSIFICATION OF $d=3$ TOPOLOGICAL INSULATORS (SUPERCONDUCTORS)}

In this section we review the classification of $d=3$ topological insulators (superconductors). This provides the 


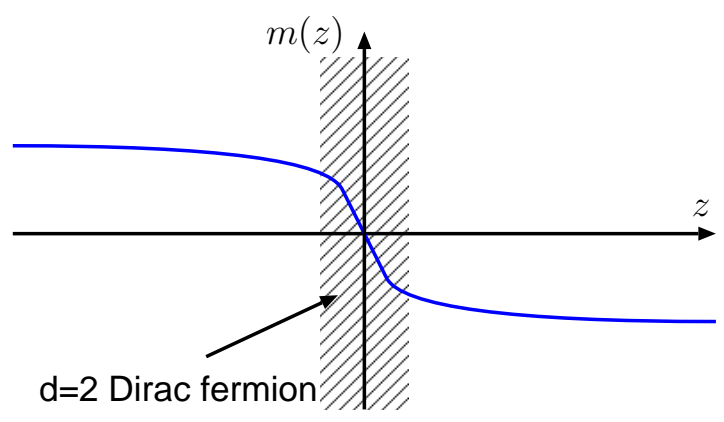

FIGURE 5. Domain wall arising form the change of sign of Dirac mass term.

main result of the work in [1], namely the last column of Table 2. Our approach is the one already mentioned in the Introduction (Section A). We focus on the robustness of the gapless boundary (surface) degrees of freedom: for every topological $d=3$ bulk insulator (superconductor) in one of the ten symmetry classes of Hamiltonians, listed in Table 1, there appear gapless degrees of freedom at its boundaries. These gapless boundary degrees of freedom cannot be gapped or localized by any perturbations or deformations of the Hamiltonian, whether these are (i) spatially uniform or whether they (ii) break translational invariance (i.e., are "random"), as long as these perturbations preserve the symmetries of the given symmetry class (Table 1). Our approach thus consists in going through the ten symmetry classes of Hamiltonians in Table 1 , in $d=2$ dimensions (describing the boundary degrees of freedom), and checking whether localized or gapped boundary degrees of freedom are possible or not in each class. If localized or gapped boundary degrees of freedom are not possible, then there exists a $d=3$ topological insulator (superconductor) in this symmetry class (possessing these gapless boundary degrees of freedom).

In this analysis one needs to recognize the importance of one extra ingredient: it is well known that $d=3$ massive Dirac Hamiltonians possess topological properties; more specifically, when changing the sign of the Dirac mass term by letting that mass vary between positive and negative values, say, in one direction (e.g., in the $z$ direction, so that the mass is $m(z)$ as sketched in Fig. 5), a gapless $d=2$ Dirac fermion degree of freedom will appear at the "domain wall" where the mass goes through zero [27, 28, 29]. This shows that in general one needs to allow for the Hamiltonian of the boundary degrees of freedom to be of Dirac form (we will discuss this shortly in somewhat more detail below). This is important because it was recently demonstrated by Bernard and LeClair [30] that there are exactly 13 and not just 10 symmetry classes of Dirac Hamiltonians in $d=2$ dimensions. This is due to the fact that a $d=2$ Dirac Hamiltonian has a special $2 \times 2$ block structure (see 8 below), allowing for a "fine structure" of the general classification of Table 1 Bernard and LeClair's result thus means that some of the ten symmetry classes from Table 1 subdivide into subclasses, and this is important for our discussion.

In short, a $d=2$ Dirac Hamiltonian is of the form

$$
\mathscr{H}=\left(\begin{array}{cc}
\mathbf{V}_{+}+\mathbf{V}_{-} & -i \frac{\partial}{\partial z} \mathbf{1}+\mathbf{A}_{+} \\
+i \frac{\partial}{\partial z} \mathbf{1}+\mathbf{A}_{-} & \mathbf{V}_{+}-\mathbf{V}_{-}
\end{array}\right)
$$

where $z=x+i y, \bar{z}=x-i y$ represent the $d=2$ dimensional spatial coordinates, and $\mathbf{A}_{+}^{\dagger}=\mathbf{A}_{-}$, and $\mathbf{V}_{ \pm}^{\dagger}=\mathbf{V}_{ \pm}$are $M \times M$ matrices, which are in genera 15 functions of $(x, y)$ [here $\mathbf{1}$ is the unit matrix]. We will refer to $M$ as the number of flavors of $d=2$ Dirac fermions.

Now, the findings of Bernard and LeClair [30] are easy to state 16 in $d=2$ dimensions there are 13 symmetry classes of Dirac Hamiltonians (8) because the three symmetry classes AIII, DIII and CI from Table 1 subdivide each into two subclasses.

Since it may be useful for some readers to see the relevance of the Bernard-LeClair classification in the case of the "quantum spin Hall", or " $Z_{2}$-topological insulator" in $d=3$ dimensions [example (iii) from the Introduction, Section A], we will briefly review this connection in the following subsection. In the subsequent subsection we will discuss all other symmetry classes.

\section{D.1 $Z_{2}$-topological insulator in $d=3(d=3$ version of the "quantum spin Hall" state)}

The work of Bernard and LeClair [30] tells us ${ }^{17}$ that there exists a $d=2$ Dirac Hamiltonian in symmetry class AII (Table 1) with only a single flavor $M=1$ (in general, an odd number $M$ ) of Dirac fermions. It is known that a single flavor cannot be realized in a $d=2$ lattice model (due to the familiar "fermion doubling" phenomenon). Therefore, this situation must correspond to the boundary of a $d=3$ topological insulator in symmetry class AII. Recall that this symmetry class refers to the presence of a time-reversal symmetry whose (antiunitary) time-reversal operator squares to -1 , and is relevant for systems possessing spin-orbit coupling. Indeed, a single flavor Dirac fermion was constructed explicitly by Fu, Kane, and Mele [12] at the $d=2$ boundary of a three-dimensional (quantum spin Hall) $Z_{2}$ topological insulator. Bernard and LeClair show that the most general $d=2$ Dirac Hamiltonian with a single flavor is a $2 \times 2$

\footnotetext{
15 for non-homogeneous ("random") systems.

16 For more details see the third column of Table III in Ref. [1].

17 See, e.g., third column of Table III in Ref. [1], or Eq. (2.19) of [30].
} 
TABLE 3. Subdivision of Symmetry Classes AII, DIII and CI for $d=2$ Dirac Hamiltonians.

\begin{tabular}{|c||c|c|c|c||c|c|}
\hline Cartan nomenclature & TRS & PHS & SLS & Bernard-LeClair & $M=\#$ of fermion species \\
\hline AIII & 0 & 0 & 1 & $\begin{array}{c}(\text { AIII })_{o} \\
(\mathrm{AIII})_{e}\end{array}$ & $\begin{array}{c}(2 m-1) \\
2 m\end{array}$ \\
\hline DIII & -1 & +1 & 1 & $\begin{array}{c}(\mathrm{DIII})_{o} \\
(\mathrm{DIII})_{e}\end{array}$ & $\begin{array}{c}(2 m-1) \\
2 m\end{array}$ \\
\hline CI & & +1 & -1 & 1 & $\begin{array}{c}(\mathrm{CI})_{o} \\
(\mathrm{CI})_{e}\end{array}$ & $\begin{array}{c}(2 m-1) \cdot 2 \\
(2 m) \cdot 2\end{array}$ \\
\hline
\end{tabular}

matrix of the form

$$
\mathscr{H}=(-i)\left[\sigma_{x} \partial_{x}+\sigma_{y} \partial_{y}\right]+V(x, y) \mathbf{1},
$$

where $V(x, y)$ is a "scalar potential". It has long been known [29, 31] that this Hamiltonian lies in symmetry class AII of Table 1 Recent work established that this Hamiltonian cannot lead to localized states [32, 33, 34, 35]: indeed, in the presence of a random scalar potential $V(x, y)$ the system behaves at large length scales always like a simple diffusive metal.

\section{D.2 Topological insulators (superconductors) in $d=3$ : all cases}

In the previous subsection we have seen that there exists a topological insulator in symmetry class AII in $d=3$ dimensions, because the boundary degrees of freedom cannot be localized. In the presence of the only possible disorder potential in this symmetry class, the system becomes the simplest possible disordered metallic conductor.

Let us now turn our attention to the three symmetry classes AIII, DIII and CI which, as already mentioned above, subdivide into two subclasses each, when $d=2$ Hamiltonians with a Dirac structure (8) are considered. As summarized in Table 3 these two subclasses simply correspond to whether the number $M$ of flavors is even or odd. (More precisely this is the case for the two symmetry classes AIII and DIII; on the other hand, for the time-reversal invariant Hamiltonians in class CI, the number of flavors $M$ is an even or an odd number of Kramers doublets, so that $M=(2 n-1) \cdot 2$ or $=2 n \cdot 2$.) As it turns out, the symmetry constraints in the special symmetry classes $(\mathrm{AIII})_{o},(\mathrm{CI})_{o}$, and $(\mathrm{DIII})_{o}$ force [30] both potentials $\mathbf{V}_{ \pm}$in (8) to vanish identically. Only the potentials $\mathbf{A}_{ \pm}$can be non-vanishing: these, on the other hand, are nothing but non-Abelian gauge potentials in the three classical groups (unitary, orthogonal, and symplectic, for $(\mathrm{AIII})_{o},(\mathrm{DIII})_{o}$, and $(\mathrm{CI})_{o}$, respectively).
Let us summarize this result:

$$
\begin{gathered}
U(2 m-1) \\
S O(2 m-1) \\
S p[2(2 m-1)]
\end{gathered} \text { for } \begin{gathered}
(\mathrm{AIII})_{o} \\
(\mathrm{DIII})_{o} \\
(\mathrm{CI})_{o}
\end{gathered}
$$

Now, the important physical consequence of the fact that the symmetries in classes $(\mathrm{AIII})_{o},(\mathrm{DIII})_{o}$, and $(\mathrm{CI})_{o}$, allow only for the presence of gauge potentials is that such potentials, whether homogeneous or random, cannot (see, e.g., [29, 36, 37, 38, 39]) localize or gap out the Dirac fermions (which are certainly gapless in the absence of any potentials). The behavior of $d=2$ Dirac fermions in the presence of these random potentials is a well-studied problem (see, e.g., [29, 36, 37, 38, 39]): even though disorder may lead to highly non-trivial and interesting behaviol 18 , the value of the longitudinal surface conductivity 19 is unchanged by this type of disorder. This means that irrespective of the presence of disorder, the longitudinal surface conductivity $\sigma_{x x}$ is $\frac{1}{\pi}\left(e^{2} / h\right)$ times the number of Dirac fermion flavors $M$. (In the case of spin- or thermal conductivity, $\sigma_{x x}^{s p i n}$ or $\kappa_{x x} / T$, the conductance unit $\left(e^{2} / h\right)$ has of course to be replaced by the corresponding unit [1].) The number $M$ of Dirac fermion flavors is directly related to the integer-valued topological "winding number" $v$, discussed in (6) in the spatially homogeneous case. Thus, the longitudinal surface conductance is a direct measure of the topological index of the bulk of the topological insulator (superconductor) in these symmetry classes.

It remains to discuss symmetry class CII. One can show [1] that in this class of Dirac Hamiltonians neither spatially homogeneous nor inhomogeneous (random) potentials can gap out or localize the $d=2$ surface degrees of freedom if the number of Dirac fermion species is an odd multiple of two, corresponding to an odd number of Kramers doublets (this class possesses

18 see, e.g., [22] for the example of the topological superconductor in symmetry class CI; Ref. [22] includes also a corresponding brief discussion of the cases AIII and DIII.

19 thermal $\left(\kappa_{x x} / T\right)$ or, if $\mathrm{SU}(2)$ spin rotation symmetry is preserved by the Hamiltonian, spin conductivity $\sigma_{x x}^{s p i n}$ for superconductors. 
time reversal symmetry). One can also show that this corresponds only to a $Z_{2}$ classification, corresponding to $M=0$ (topologically trivial) and $M=2$ (topologically non-trivial); changing $M$ by four (i.e., by two Kramers doublets) does not lead to a topologically different state.

Finally, it is very easy to see [1] from the BernardLeClair classification that in all the remaining symmetry classes of the 10 classes (i.e., in classes A, AI, BDI, D, C) the $d=2$ Dirac Hamiltonian can be made fully gapped while keeping all defining symmetries of the class intact. By performing these steps, we arrive at the results listed in the last column (entitled " $d=3$ ") of Table 2 This is our main result, obtained in [1].

\section{E. CLASSIFICATION OF $d=2$ TOPOLOGICAL INSULATORS (SUPERCONDUCTORS)}

We briefly summarize from [1] the classification of $d=2$ topological insulators (superconductors). It may be useful for the reader to follow the discussion by keeping an eye on Table 2. There are three well known symmetry classes which support topological insulators (superconductors) in $d=2$ : these are symmetry classes $\mathrm{A}, \mathrm{D}$, and $\mathrm{C}$, all of which break time-reversal symmetry, and are all known [2, 40, 41, 42, 43] to possess a quantum Hall insulating state. The latter manifests itself by the appearance of chiral edge states. Classes A and D were discussed in examples (i) and (ii) in the Introduction (Section A), and class $\mathrm{C}$ is known as the so-called spin quantum Hall effect [42, 43] (not to be confused with the "quantum spin Hall state" discussed in example (iii) of the Introduction). Since these states may possess any number of chiral edge states, the different topological sectors of the $d=2$ insulators (superconductors) are characterized by integers. This is the origin of the entries $\mathbf{Z}$ in the penultimate column of Table 2] This same column contains in addition an entry $Z_{2}$ in the row labeled AII: this is the $d=2$ "quantum spin Hall" insulator discussed in example (iii) of the Introduction. It remains to discuss the row labeled DIII. This case was treated in [44], where the authors studied the localization physics of (quasi-) onedimensional systems: the authors found that a (quasi-) one-dimensional Hamiltonian in symmetry class DIII cannot be localized or gapped if there is an odd number of one-dimensional modes. This situation can be realized [1, 45, 46] in chiral p-wave superconductors with opposite chiralities $\left[\left(p_{x}+i p_{y}\right)\right.$ and $\left(p_{x}-i p_{y}\right)$ pairing symmetries]. Moreover, in the remaining five symmetry classes of Table 2, (quasi-) one-dimensional Hamiltonians will always generically be localized or gapped. This is related, in great generality, to the well-known " 12 -fold way" classification scheme 20 of random transfer matrices, summarized in Table IV of [1].

\section{F. CLASSIFICATION OF $d=1$ TOPOLOGICAL INSULATORS (SUPERCONDUCTORS)}

Again, we proceed [1] as for dimensions $d=2$ and $d=3$, reviewed above: the diagnostic of a $d=1$ topological insulator (superconductor) is the appearance of gapless degrees of freedom at the boundaries. In $d=1$ the boundaries are points. Thus, we need to check in which of the 10 symmetry classes of Table 1 gapless degrees of freedom ("zero modes") appear at a point. The answer to this question is known from random matrix theory 21 , and was found for all 10 symmetry classes in 2001 by D. Ivanov [47]. A summary of these results is displayed in Table V of [1]. Using this information, one arrives at the column entitled " $d=1$ " of Table 2 .

\section{G. DISCUSSION}

Table 2 summarizes the main result of this work, the classification of topological insulators (superconductors) in spatial dimension $d=1,2$, and 32 The symmetry classes in Table 2 are organized according to the physical systems these symmetry classes represent (three Wigner-Dyson classes of standard electronic systems; three Wigner-Dyson classes with extra ("sublattice" or

\footnotetext{
20 Two of the ten symmetry classes undergo a subdivision; these are precisely the symmetry classes AII and DIII where $Z_{2}$ topological insulators exists in $d=2$. The existence of these topological states is precisely related to this splitting. This is similar to what happened in the Bernard LeClair classification scheme [30] for $d=2$ Dirac Hamiltonians, discussed in Section D.

21 describing Hamiltonians in spatial dimension $d=0$

22 One can understand the presence of the key signatures of topological insulators, namely, the stability of their gapless nature and the complete absence of Anderson localization for boundary degrees of freedom, using a variety of techniques and from different points of views. The following presents yet another slightly different way of thinking about this. The Anderson localization problem at the boundary of topological insulators can also be discussed in terms of the NLSM formalism in a rather unified fashion. (We may choose here the so-called "fermionic replica" formulation (see the last column in Table 2, but we may, equivalently, choose the formulation using supersymmetry [23].) When the NLSM formalism is applied to describe effects of disorder on the gapless boundary degrees of freedom, the fact that these boundary degrees of freedom completely evade Anderson localization is signaled by an additional term which can be added to the NLSM action. Depending on the symmetry class and spatial dimensions, it takes on the form of either a topological or a Wess-Zumino-Witten (WZW) term. In turn, the presence or absence of a topological or WZW term for a given symmetry class in $d$ dimensions can be read off from Bott periodicity - see below. (Compare also footnote 9.)
} 
TABLE 4. Reorganizing Table 2 by reordering the symmetry classes and grouping them into two separate lists reveals a regular pattern, which was recently pointed out by A. Kitaev Ref. [16].

\begin{tabular}{|c|c|c|c|c|c|c|c|}
\hline Cartan nomenclature & TRS & PHS & SLS & Hamiltonian & $d=1$ & $d=2$ & $d=3$ \\
\hline AIII (chiral unit.) & 0 & 0 & 1 & $U(N+M) / U(N) \times U(M)$ & $\mathbf{Z}$ & - & $\mathbf{Z}$ \\
\hline A (unitary) & 0 & 0 & 0 & $U(N)$ & - & $\mathbf{Z}$ & - \\
\hline BDI (chiral orthog.) & +1 & +1 & 1 & $S O(N+M) / S O(N) \times S O(M)$ & $\mathbf{Z}$ & - & - \\
\hline $\mathrm{D}$ & 0 & +1 & 0 & $S O(2 N)$ & $\mathbf{Z}_{2}$ & $\mathbf{Z}$ & - \\
\hline DIII & -1 & +1 & 1 & $S O(2 N) / U(N)$ & $\mathbf{Z}_{2}$ & $\mathbf{Z}_{2}$ & $\mathbf{Z}$ \\
\hline AII (symplectic) & -1 & 0 & 0 & $U(2 N) / S p(2 N)$ & - & $\mathbf{Z}_{2}$ & $\mathbf{Z}_{2}$ \\
\hline CII (chiral sympl.) & -1 & -1 & 1 & $S p(2 N+2 M) / S p(2 N) \times S p(2 M)$ & $\mathbf{Z}$ & - & $\mathbf{Z}_{2}$ \\
\hline $\mathrm{C}$ & 0 & -1 & 0 & $S p(2 N)$ & - & $\mathbf{Z}$ & - \\
\hline $\mathrm{CI}$ & +1 & -1 & 1 & $S p(2 N) / U(N)$ & - & - & $\mathbf{Z}$ \\
\hline AI (orthogonal) & +1 & 0 & 0 & $U(N) / O(N)$ & - & - & - \\
\hline
\end{tabular}

"chiral" symmetry: $\mathrm{SLS}=1$ ); four classes of BdG Hamiltonian in superconductors). While such an ordering is natural from the physics point of view, it hides an underlying mathematical structure, namely a periodicity in spatial dimension $d$, which was recently pointed out by Kitaev [16]. To uncover this periodicity we have reorganized Table 2 by reordering the symmetry classes and grouping them into two separate lists (see Table 4). The upper list in Table 4 contains only the two unitary classes A and AIII, both of which have neither TRS nor PHS (discussed in Sec. B). These two classes are related to the two types of classifying spaces appearing in complex K-Theory of Ref. [16]. We see from the reordered Table 4 that an alternating pattern (period 2) in the spatial dimension $d$ becomes apparent: i.e., the class AIII topological insulator can only exist in odd spatial dimensions, while the class A topological insulator (i.e., the integer quantum Hall insulator) occurs only in even spatial dimensions. The lower list in Table 4 (classes BDI, D, $\cdots$, $\mathrm{AI})$ contains all the remaining classes; those are the ones that have at least either TRS or PHS. These eight classes are related to the eight types of classifying spaces appearing in real K-Theory, discussed in Ref. [16]. An obvious regular pattern emerges when looking at the reordered Table 4 as the spatial dimension $d$ is increased by one, the topological insulators (superconductors) move down by one column. It was shown by Kitaev that this regular pattern is due to an 8-fold periodicity in $d$, the Bott periodicity of real K-theory. Taking this result from K-theory, we can extend our result to dimensions $d>3$. For example, Table 4 suggests that in $d=4$ there is a topological insulator whose topologically distinct sectors are classified by integers $\mathbf{Z}$, which belongs to symmetry class AII. Indeed, Qi et al. [48] have recently shown that the $Z_{2}$ topological insulators of the class AII in $d=2$ and 3 can be obtained as descendants from this four-dimensional $Z$ topological insulator using dimensional reduction.

\section{ACKNOWLEDGMENTS}

We thank Alexei Kitaev for stimulating discussions. This work was supported in part by the National Science Foundation (NSF) under Grant Numbers PHY0551164 (S.R., A.S., A.F.) and DMR-0706140 (A.W.W.L.). A.W.W.L. thanks the organizers of the Landau Memorial Conference "Advances in Physics" for the opportunity to present this work.

\section{REFERENCES}

1. Andreas P. Schnyder, Shinsei Ryu, Akira Furusaki, and Andreas W. W. Ludwig, Phys. Rev. B 78, 195125 (2008); see also "Anderson Localization at Boundaries: Classification of Topological Insulators and Superconductors", http://landau100.itp.ac.ru/Talks/ludwig.pdf

2. N. Read and D. Green, Phys. Rev. B 61, 10267 (2000).

3. E. Witten, Commun. Math. Phys. 121, 351 (1989).

4. G. Moore and N. Read, Nucl. Phys. B 360, 362 (1991).

5. Y. Hatsugai, Phys. Rev. Lett. 7, 3697 (1993).

6. E. Witten, arXiv:0706.3359 (unpublished).

7. C. L. Kane and E. J. Mele, Phys. Rev. Lett. 95, 146802 (2005); 95, 226801 (2005). B. A. Bernevig and S.-C. Zhang, Phys. Rev. Lett. 96, 106802 (2006).

8. M. König, H. Buhmann, L. W. Molenkamp, T. Hughes, C.-X. Liu, X.-L. Qi, and S.-C. Zhang, J. Phys. Soc. Jpn. 77, 031007 (2008).

9. M. König, S. Wiedmann, C. Brune, A. Roth, H. Buhmann, L. Mohlenkamp, X.-L. Qi, and S.-C. Zhang, Science 314, 1757 (2006).

10. J. E. Moore and L. Balents, Phys. Rev. B 75, 121306(R) (2007).

11. Rahul Roy, arXiv:cond-mat/0607531 (unpublished). 
12. L. Fu, C. L. Kane, and E. J. Mele, Phys. Rev. Lett. 98, 106803 (2007).

13. D. Hsieh, D. Qian, L. Wray, Y. Xia, Y. Hor, R. Cava, and M. Hasan, Nature 452, 970 (2008)

14. D. Hsieh, Y. Xia, L. Wray, D. Qian, A. Pal, J. H. Dil, J. Osterwalder, F. Meier, G. Bihlmayer, C. L. Kane, Y. S. Hor, R. J. Cava, and M. Z. Hasan, Science 323, 919 (2009).

15. M. R. Zirnbauer, Phys. Rev. Lett. 69, 1584 (1992); A. D. Mirlin, A. Müller-Groeling, and M. R. Zirnbauer, Ann. Phys. (N.Y.) 236, 325 (1994).

16. A. Yu. Kitaev, "Periodic table for topological insulators and superconductors", this Volume; arXiv:0901.2686, see also "Periodic Table for Topological Insulators and Superconductors", http://landau100.itp.ac.ru/Talks/kitaev.pdf

17. M. R. Zirnbauer, J. Math. Phys. 37, 4986 (1996).

18. A. Altland and M. R. Zirnbauer, Phys. Rev. B 55, 1142 (1997).

19. See, e.g., also a more recent treatment: P. Heinzner, A. Huck Leberry, and M. R. Zirnbauer, Commun. Math. Phys. 257, 725 (2005).

20. F. J. Dyson, J. Math. Phys. 3, 1199 (1962); M. L. Mehta, Random Matrices, Academic Press, Boston, 1991.

21. G. E. Volovik, in The Universe in a Helium Droplet, The International Series of Monographs on Physics Vol. 117, Oxford University Press, New York, 2003; G. E. Volovik, in Exotic Properties of Superfluid 3He, Series I N Modern Condensed Matter Physics Vol. 1, World Scientific, Singapore, 1992.

22. Andreas P. Schnyder, Shinsei Ryu, and Andreas W.W. Ludwig, arXiv:0901.1343 (unpublished).

23. K. Efetov, Supersymmetry in Disorder and Chaos, Cambridge University Press, Cambridge, England, 1997.

24. B. DeWitt, Supermanifolds, Cambridge University Press, Cambridge, England, 1992.

25. M. Kohmoto, B. I. Halperin, and Y. S. Wu, Phys. Rev. B 45, 13488 (1992).

26. A. Pruisken, Nucl. Phys. B 235, 277 (1984).

27. C. G. Callan, Jr. and J. A. Harvey, Nucl. Phys. B 250, 427 (1985).

28. F. D. M. Haldane, Phys. Rev. Lett. 61, 2015 (1988).

29. A. W. W. Ludwig, M. P. A. Fisher, R. Shankar, and G. Grinstein, Phys. Rev. B 50, 7526 (1994).

30. D. Bernard and A. LeClair, J. Phys. A 35, 2555 (2002).

31. T. Ando and T. Nakanishi, J. Phys. Soc. Jpn. 67, 1704 (1998); T. Ando, T. Nakanishi, and R. Saito, ibid. 67, 2857 (1998).

32. S. Ryu, C. Mudry, H. Obuse, and A. Furusaki, Phys. Rev. Lett. 99, 116601 (2007).

33. K. Nomura, M. Koshino, and S. Ryu, Phys. Rev. Lett. 99, 146806 (2007).

34. P. M. Ostrovsky, I. V. Gornyi, and A. D. Mirlin, Phys. Rev. Lett. 98, 256801 (2007).

35. J. H. Bardarson, J. Tworzydlo, P. W. Brouwer, and C. W. J. Beenakker, Phys. Rev. Lett. 99, 106801 (2007).

36. A. M. Tsvelik, Phys. Rev. B 51, 9449 (1995).

37. C. Mudry, C. Chamon, and X.-G. Wen, Nucl. Phys. B 466, 383 (1996).

38. M. J. Bhaseen, J.-S. Caux, I. I. Kogan, and A. M. Tsvelik, Nucl. Phys. B 618, 465 (2001).

39. A. W. W. Ludwig, arXiv:cond-mat/0012189 (unpublished).
40. R. Prange, S. Girvin, The Quantum Hall Effect, Springer Verlag, Berlin, Germany, 1990.

41. T. Senthil and Matthew P. A. Fisher, Phys. Rev. B 61, 9690 (2000); N. Read and A. W. W. Ludwig, Phys. Rev. B 63, 024404 (2001); J. T. Chalker, N. Read, V. Kagalovsky, B. Horovitz, Y. Avishai, and A. W. W. Ludwig, Phys. Rev. B 65, 012506 (2002).

42. T. Senthil, J. B. Marston, and M. P. A. Fisher, Phys. Rev. B 60, 4245 (1999); Ilya A. Gruzberg, Andreas W. W. Ludwig, and N. Read, Phys. Rev. Lett. 82, 4524 (1999).

43. T. Senthil, M. P. A. Fisher, L. Balents, and C. Nayak, Phys. Rev. Lett. 81, 4704 (1998).

44. I. A. Gruzberg, N. Read, and S. Vishveshwara, Phys. Rev. B 71, 245124 (2005).

45. R. Roy, arXiv:cond-mat/06008064 (unpublished); arXiv:0803.2868 (unpublished)

46. X.-L. Qi, T. L. Hughes, S. Raghu, and S.-C. Zhang, arXiv:0803.3614 (unpublished).

47. D. A. Ivanov, arXiv:cond-mat/9911147 (unpublished); D. A. Ivanov, J. Math. Phys. 43, 126 (2002).

48. X.-L. Qi, T. Hughes, and S.-C. Zhang, Phys. Rev. B 78, 195424 (2008). 